\title{
Biomechanics Analysis of Passing Accuracy by Using Foot and Kick Distance at the Student Football Player
}

\author{
Komarudin $^{1}$, Sugiharto ${ }^{2}$, Hari Setijono ${ }^{3}$, Setya Rahayu ${ }^{4}$ \\ ${ }^{1}$ Faculty of Sport Sciences, Universitas Negeri Yogyakarta, Indonesia \\ ${ }^{2,4}$ Graduate School, Universitas Negeri Semarang, Indonesia \\ ${ }^{3}$ Graduate School, Universitas Negeri Surabaya, Indonesia \\ ${ }^{1}$ Corresponding author: komarudin@uny.ac.id
}

\begin{abstract}
Football is one kind of invasion game. Recently, football become more popular around the world. The purpose of this study was 1) to obtain biomechanics analysis, understanding the factors that affect the accuracy of the target when student football player passing the ball based on using foot and distance, and 2) to obtain optimal combination between those factors. We performed an experiment in which 15 student football player from Center of Education and Training Sport Program Central Java Province of Indonesia to participated. Their average mass was $(63 \pm 13.19) \mathrm{kg}$ and average height was $(172 \pm 6.23) \mathrm{cm}$. The experiment was designed by using two -way factorial design. Each respondent passing the ball against to the target with four different condition based on using foot (inside foot and instep foot) and distance (10 meters and 20 meters from the target). Plaform 1 in AMTI Biomechanics Force Platforms BP400600H was used to collect the kinematics data from Ground Reaction Force (GRF) and Required Coefficient of Friction (RCOF). The results showed that 1) distance factor influence significantly to the accuracy of the target with $\mathrm{p}$ value $=0.001$ and 2 ) the interaction between those factors by using foot and distance also have a significant result to the GRF with $\mathrm{p}$ value $=0.005$.
\end{abstract}

Keywords: Biomechanics Analysis; Passing Accuracy; Foot Using and Kick Distance

\section{Introduction}

Football is one type of sports game. Today, football is becoming very popular throughout the world (Fabio, Lilian, Paulo, \& Sergio, 2010). FIFA (Fédération Internationale de Football Association) does not doubt that football will continue to develop so that it becomes an important and interesting part of football (FIFA, 2004). This development is due to the increasing demand for information related to football, so as to provide a better understanding to the public about football, especially in terms of the quality of the game and its ins and outs (Moore, Bullough, Goldsmith, \& Edmondson, 2014).

In playing football there are a number of basic techniques that must be mastered by players, namely dribbling techniques, kicking techniques, and passing techniques. The technique of passing the ball is part of the technique of kicking the ball in football games. Kicking a ball is important during an attack in a football game, and a team with more accurate shots against the target will get a better chance of scoring goals and winning games (Kellis and Katis, 2007). Kick variations become an important factor as a football player by combining slow, medium and tight kicks during a game (Ismail, Adnan, \& Sulaiman, 2014).
There are two ways a football player performs kicking techniques, namely kicking using the inner legs and kicking using the back of the foot. Kicking using an inner leg is usually used by football players to pass the ball at close range during the game. Meanwhile, football players who kick using the back of the foot are usually used to pass the ball far and near and are used for penalty kicks and shooting at the goal (Ismail, Adnan, \& Sulaiman, 2014).

Ball passing activity is an important activity in football games. Because to put the ball into the opponent's goal, a team must develop a strategy by making accurate passes to each player before making a kick to put the ball into the opponent's goal. Inaccurate operands will cause a team to lose the chance to put the ball into the opponent's goal.

Passing is the basis of a football game that must be mastered and is needed in football games. Because, with a small field area and flat field contours, it takes hard, fast, and accurate operands. Almost all football games are always used to pass the ball. To have good operability or passing skills, mastery of the movement is needed so that the desired target to be passed is achieved accurately (Hermans, V \& Engler, R., 2011).

Seeing the importance of the accuracy of ball passes in football games, research is needed to improve the performance of football players 
when passing the ball. The optimal combination of the distance of the target and the use of the foot when passing the ball uses the inner foot and back of the foot to the accuracy of the pass, in this case limited by student football player from Center of Education and Training Sport Program Central Java Province of Indonesia to participated can be performed to improve the performance of football players when passing the ball. Where by knowing this, the best combination of accurate ball passes can be known, so that a biomechanical analysis of football players can be obtained when passing the ball accurately.

\section{Methods}

This experimental design was made involving 15 respondents, where this respondent was a football player from Center of Education and Training Sport Program Central Java Province of Indonesia aged 15 to 18 years old. The selected respondents weigh $63 \pm 13.19 \mathrm{~kg}$ and height $172 \pm 6.23 \mathrm{~cm}$. The selection of respondents' criteria is based on the size of body weight and height of the population body in Indonesia which is still included in the 50th percentile. In the study it is known that the number of football players who are members of the Center of Education and Training Sport Program Central Java Province of Indonesia is 22 people, the sample taken in this study is 15 people because the other 7 respondents are not included in the criteria of the selected respondents namely those who weigh $63 \pm 13.19$ $\mathrm{kg}$ and height $172 \pm 6.23 \mathrm{~cm}$.

The tool used in this study is the AMTI Biomechanics Force Platforms BP400600HF which is a tool used to detect forces and moments that affect the surface of the platform. In this study, only platform 1 is used because when the respondent passes the ball, the foot that rests on the plane is only one foot. The dimensions of platform 1 are $400 \mathrm{~mm} \times 600 \mathrm{~mm}$ $\mathrm{x} 82.5 \mathrm{~mm}$. This platform is operated at a frequency of $1000 \mathrm{~Hz}$. Meanwhile, the devices used in this study were two pairs of football shoes with a size of 40 and 43 respectively, one football ball, and a target beam that had been given a number to measure accuracy.

There are two factors that will be tested in this experiment, namely the factor of foot use and target distance when the player passes the ball. Each respondent uses footwear that matches the respondent's foot size, so it does not affect the performance of the respondent when passing the ball. Each respondent passes a pass to the target with the combination that has been designed according to the method in the Design of Experiment, which is Two Level Factorial Design for each factor and level.

There are two objects in this data retrieval, the first is data retrieval using the target beam as the target to measure accuracy and the second is data retrieval using a force plate to measure the amount of Ground Reaction Force (GRF).

The first data retrieval uses the target beam as a target to measure accuracy. Accuracy is measured by the way each respondent passes the ball according to a predetermined combination with the target of the center point of the target beam. Inside the target beam there are numbers that indicate the accuracy parameters. The parameters used are numbers, the numbers used start from 1 to 10 on the left end to midpoint and 10 to 1 at the midpoint to the right end with the number 10 as the midpoint. The more the ball hits the target midpoint of the beam, the more accurate the pass will be made by the respondent. In this way, an optimal combination of passing the ball will be obtained with the factors and levels determined in the experimental design.

The second data retrieval uses a force plate, and only platform 1 is used to retrieve data because when the respondent passes the ball, the foot that rests on the plane is only one foot. In this study, limited respondents used to the right foot to pass the ball. So, the foot that supports the platform 1 is the respondent's left foot. When the respondent passes the ball, the left foot of the respondent stepping on platform 1 will produce a compressive force on platform 1 and will later produce Ground Reaction Force (GRF) which is the same size as the respondent's compression force on platform 1. So, the GRF data will be recorded in real time on force plate.

The following is the total number of respondents experimenting with four kinds of combinations of circumstances:

Passing the ball using an inner foot with a distance of 10 meters.

1) Passing the ball using a foot with a distance of 20 meters.

2) Passing the ball using an inner foot with a distance of 10 meters.

3) Passing the ball using a foot with a distance of 20 meters. 


\section{Data collection}

Data collection in this study is primary data collection, because data is collected directly. These data collected directly include target accuracy data when the respondent passes the ball. Then proceed with the collection of large data Ground Reaction Force (GRF) and the amount of Required Coefficient of Friction (RCOF) which is measured using a force plate.

\section{Results and Discussion Biomechanics}

Biomechanics is a multidisciplinary science that involves the application of mechanical principles in the study of the structure and function of living organisms. Statics and dynamics are the two main branches of mechanics (Hall, 2012). Statics is the study of systems that are located in a state of constant motion, that is, either at rest (in the absence of movement) or moving at a constant speed. Dynamics is the study of systems that are accelerating.

Kinematics and kinetics are also subdivisions of studies on biomechanics. Kinematics involves the study of the size, sequence, and time of movement, without referring to the force that causes or results from the movement. Furthermore, kinetics is the study of forces associated with motion. Style can be considered as an impulse or attraction about the body so that the study of style is usually a kinetics study. The study of biomechanics in humans may also answer questions such as how much force on the muscles is optimal to produce a movement.

Biomechanics of the human movement is a branch of kinesiology. In addition, biomechanics is also a scientific branch of the science of sports medicine. Sport medicine is a general term that covers clinical and scientific aspects of sports.

\section{Ground Reaction Force (GRF)}

Ground reaction force is a compressive force that is proportional and opposite in direction to the compressive force that is given by the body above the surface of the fulcrum through the foot (Novlinda, 2012). Measurement of Ground Reaction Force (GRF) is to use a force platform placed in the middle of the track as a foot support when kicking. The amount of Ground Reaction Force (GRF) is measured in three components, namely vertical force $(\mathrm{Fz})$, lateral force $(\mathrm{Fx})$, and med-lateral $(\mathrm{Fy})$ force.
Each component produces different GRF. Research on GRF for medical and sports applications has been investigated (Winter, 1990); (Inman, et al., 1981); (Perry, 1992).

\section{Required Coefficient of Fiction (RCOF)}

There are many cases of accidents that occur, both at home and at work where the cause of this accident is when humans fall. The second most common cause of accidents is slipping, which is $25 \%$.

Because of the high incidence of humans falling and slipping, a lot of research has been done on the coefficient of friction. In order for someone not to slip, the Available Coefficient of Friction (ACOF) must be greater than the Required Coefficient of Friction (RCOF). The greatest RCOF value when a human walks normally is during the contact phase with the touch plane surface.

\section{Processing of Accuracy, GRF and RCOF Data}

After data collection of respondents, researchers used Minitab 16 software's statistical tools, namely Design of Experiment with features, namely Analyze Factorial Design to process accuracy, GRF, and RCOF data in the form of residual probability plots before proceeding with ANOVA data processing.

The residual probability plots for processing accuracy, GRF, and RCOF data are normally distributed. Judging from the p-value, the accuracy, GRF, and RCOF are greater than the values set in this data processing, namely (= $0.05)$. The $p$-value values of each of these factors are $0.125,0.374$ and 0.525 respectively.

\section{ANOVA Processing Accuracy, GRF and RCOF data}

Once it is known that the residual probability plot for processing accuracy, GRF, and RCOF data is normally distributed. Furthermore, it will be seen the significance, the level of significance seen is from independent factors, namely (foot and distance use) on dependent factors, namely (accuracy, GRF, and RCOF) when doing ball passes. ANOVA processing results from the accuracy, GRF, and RCOF data can be seen in Table 1 below. 
Table 1. ANOVA Processing Results from Accuracy, GRF, and RCOF Data

\begin{tabular}{llll}
\hline & $\begin{array}{l}\text { Accuracy } \\
p \text {-value }\end{array}$ & $\begin{array}{l}\text { GRF } \\
p \text {-value }\end{array}$ & $\begin{array}{l}\text { RCOF } \\
p \text {-value }\end{array}$ \\
\hline Foot usage & 0.352 & 0.608 & 0.245 \\
Distance & 0.001 & 0.144 & 0.529 \\
$\begin{array}{l}\text { Foot usage } \\
\text { *Distance }\end{array}$ & 0.467 & 0.005 & 0.126 \\
\hline
\end{tabular}

From the results of data processing it can be seen that the distance factor has a statistically significant effect with $p$-value $=0.001$ in producing accuracy, and for the interaction between the two main factors namely the use of feet and distance has a statistically significant effect with $p$-value $=0.005$ in generating the magnitude of GRF.

From previous studies, no studies have been conducted on the accuracy of passing a ball. Previous studies discussed the accuracy of kicking a ball. Kick accuracy depends on how fast the player's foot touches the ball (Godik et al., 1993). When a player is ordered to take a kick using the back of the foot, the fastest kick is the most accurate kick. From this it can be indicated that there is a speed relationship in producing accurate kicks (Godik et al., 1993).

Previous studies also mentioned that good techniques can produce accurate kicks (Kellis and Katis, 2007). Ball position and distance of the player in kicking the ball affect the accuracy of the kick (Kellis and Katis, 2007). The results of this study also show that the operand distance of the player to the target has a significant effect on the magnitude of accuracy, with p-value = 0.001 smaller than that determined, namely $=$ 0.05 .

GRF from previous studies explains, that fatigue in carrying out biomechanical activities is caused by changes in GRF and kinematics during running (Mizrahi et al, 2000; Williams et al., 1991). The results are different from previous studies, in this study GRF became a dependent factor to determine the significant influence between the use of the foot and the distance of the player in passing the ball. And the results obtained are significant interactions between the use of the foot and the distance of the player in passing the ball with $p$-value = 0.005 smaller than that specified, namely $=0.05$. Previous studies have not mentioned any research regarding RCOF in making kicks or operands in playing football. In this study, RCOF was conducted to measure the magnitude of the coefficient of friction when the player passed the ball.

\section{Conclusion}

Research conducted on the activity of passing a ball using the design of this experiment was carried out to obtain an optimal combination of foot and distance use of football players when passing the ball to the accuracy of the target, and to obtain a biomechanical analysis of the ball passing activity in the form of Ground Action Force (GRF) and Required Coefficient of Friction (RCOF). The components analyzed in this study are the accuracy of ball operands, GRF, and RCOF of the respondents when conducting experiments.

Based on the results of data processing and analysis, the conclusion of the entire experiment is as follows:

1) The optimal combination of the factors using the foot and the distance of the player when passing the ball to the accuracy of the target, is when the condition uses the inner foot with a operative distance of 10 meters which results in a percentage of accuracy $(91 \pm$ 7) $\%$.

2) The amount of GRF when the player passes the ball is equal to $(959.8 \pm 149.9)$ $\mathrm{N}$.

3) The amount of RCOF when the player passes the ball is $(0.29 \pm 0.06)$.

4) The factor of the distance of the player when passing the ball has a statistically significant effect on the accuracy of the target, as evidenced by the p-value generated in data processing of 0.001 less than that specified in the experiment, which is 0.05 .

5) The interaction of factors using foot and distance of players when passing the ball statistically significant effect on the value of $\mathrm{Fz}$ (vertical force) produced, as evidenced by the p-value generated in processing data of 0.005 less than that specified in the experiment, which is 0.05 . 


\section{References}

Fabio A.B., Lilian T.B.G., Paulo R.P.S. \& Sergio A.C. (2010). Performance comparisons of the kicking of stationary and rolling balls in a football context. Sport Biomechanics, 9 (1), 1-15.

FIFA. (2004). Info Plus. Zurich: Federation Intenationale de Football Association.

Godik, M., Fales, I. and Blashak, I. (1993) Changing the kicking accuracy of football players depending on the type, value and aims of training and competitive loads. In: Science and football II. Eds: Reilly, T., Clarys, J. and Stibbe, A. London: E\&FN Spon. 254260.

Hall, S.J. (2012). Basic Biomechanics Sixth Edition. New York: Mc Graw Hill.

Hermans, V \& Engler, R. (2011). football Technique Tactics Training. New York: Meyer \& Meyer Sport.
Ismail, S.I., Adnan, R., \& Sulaiman, N. (2014). Moderate Effort Instep Kick in Sepakbola. Procedia Engineering, 186191.

Kellis, E., Katis, A. (2007). Biomechanical characteristics and desterminants of instep football kick. Journal of Sports Science and Medicine, 154-165.

Mizrahi, J., Verbitsky, O., Isakov, E. and Daily, D. (2000) Effect of fatigue on leg kinematics and impact acceleration in long distance running. Human Movement Science 19, 139- 151.

Moore R., Bullough S., Goldsmith S., \& Edmondson L. (2014). A systematic review of sepakbola literature. American Journal of Sports Science and Medicine. 2 (3), 108-116. 